\title{
Effect of nonstoichiometry on the magnetic and electrical properties of synthetic single crystal $\mathrm{Fe}_{2.4} \mathrm{Ti}_{0.6} \mathrm{O}_{4}$
}

\author{
B.J. Wanamaker and Bruce M. Moskowitz \\ Department of Geology and Geophysics and Institute for Rock Magnetism, University of Minnesota, \\ Minneapolis, Minnesota
}

\begin{abstract}
A single crystal of titanomagnetite $\mathrm{Fe}_{2,4} \mathrm{Ti}_{0.6} \mathrm{O}_{4}$ (TM60) was synthesized using the floating zone technique. The Curie temperature, saturation magnetization at $5 \mathrm{~K}$, and thermopower were measured for several pieces of the crystal as grown and following high temperature annealing at different oxygen fugacities within the stability field of the TM60. The magnetic and electrical data indicate that long-range ordering in TM60 is a function of nonstoichiometry with higher cation vacancy concentrations producing a more random cation distribution. This effect may explain the differences among cation distribution models for TM developed previously by other workers.
\end{abstract}

\section{Introduction}

The titanomagnetite (TM) solid solution series is an important mineral system in petrology and geophysics. Yet, there is still disagreement about major crystal chemical aspects of this mineral system [e.g., Lindsley, 1991]. Questions regarding the extent of nonstoichiometry and its effects on magnetic and thermodynamic properties are largely unexplored. Variations in intrinsic magnetic properties caused by changes in the crystal chemistry of titanomagnetite lead to subtle but complex changes in magnetic domain states, magnetic remanence and its stability. A complete understanding of the magnetic and thermochemical properties of titanomagnetites is essential for a proper interpretation of the paleomagnetic behavior of rocks containing this phase and its oxidized products.

Titanomagnetites $\left(\mathrm{Fe}_{3-x} \mathrm{Ti}_{x} \mathrm{O}_{4} \equiv(1-x) \mathrm{Fe}_{3} \mathrm{O}_{4} \cdot x \mathrm{Fe}_{2} \mathrm{TiO}_{4}\right)$ form a complete solid solution with the spinel structure above approximately $600^{\circ} \mathrm{C}$ between the end members magnetite $\left(\mathrm{Fe}_{3} \mathrm{O}_{4}\right)$ and ulvöspinel $\left(\mathrm{Fe}_{2} \mathrm{TiO}_{4}\right)$, although intermediate compositions may be metastably preserved to lower temperatures by quenching. In addition, titanomagnetites often deviate from ideal $4 / 3$ oxygen/cation stoichiometry by the formation of cation vacancies. At high temperatures $\left(>1000^{\circ} \mathrm{C}\right)$, TM can accommodate a finite degree of nonstoichiometry of a few percent within its stability field [Hauptman, 1974; Aragon and McCallister, 1982]. A generalized formula for the cation distribution for any nonstoichiometric titanomagnetite can be given in terms of one distribution parameter (b) and two compositional parameters $(x, \delta)\left[O^{\prime}\right.$ Reilly, 1984]

$$
\left(F e_{b}^{3+} F e_{1-b}^{2+}\right)\left[F e_{(2-2 x)+2 \delta(3+x)-b}^{3+} F e_{(b+x)-\delta(9+x)}^{2+} T i_{x(1-\delta)}^{4+} \square_{38}\right] O_{4}^{2-}
$$

where 0 and [] denote A (tetrahedral) and B (octahedral) sites respectively, $b$ is the number of $\mathrm{Fe}^{3+}$ ions on $\mathrm{A}$ sites, $\delta$ is the vacancy concentration, and $\square$ represents a cation Copyright 1994 by the American Geophysical Union.

Paper number 94GL00877

0094-8534/94/94GL-00877\$03.00 vacancy. The nonstoichiometry parameter $\delta$ increases as a function of temperature and oxygen fugacity, theoretically varying between $0<\delta<(1+x) /(9+x)$ [Dieckmann, 1982; Aragon and McCallister, 1982]. Experimental estimates for the maximum degree of nonstoichiometry at $1300^{\circ} \mathrm{C}$ for TM6070 are $\delta_{\max }=0.01-0.02$ [Hauptman, 1974; Senderov et al., 1993]. The oxide spinel model of O'Neill and Navrotsky [1984] characterizes the cation distribution for TM at stoichiometry by a distribution coefficient $K_{c d}$ for the exchange reaction $\left(F e^{2+}\right)_{A}+\left[F e^{3+}\right]_{B}=\left(F e^{3+}\right)_{A}+\left[F e^{2+}\right]_{B}$

$$
K_{c d}=\frac{\left(F e^{3+}\right)\left[F e^{2+}\right]}{\left(F e^{2+}\right)\left[F e^{3+}\right]}=\frac{b(x+b)}{(1-b)(2-2 x-b)}
$$

When $K_{c d}=0,1$, or $\infty$, the cation distribution is respectively normal $(b=0)$, random $(b=(2-2 x) /(3-x))$, or inverse $(b=1$, $x \leq 0.5 ; b=2(1-x), x \geq 0.5)$.

Although nonstoichiometry and short range ordering have been suggested before as possible explanations for the disparate magnetic results obtained on the TM series by numerous investigators [e.g., Wechsler et al., 1984; Moskowitz, 1987], there has yet to be definitive experimental confirmation of these hypotheses. Here we describe a method for synthesizing large single crystals of titanomagnetite and a series of magnetic and electrical measurements that may be used to investigate the effects of variations in point defect stoichiometry on the mineral physics of this material.

\section{Synthesis of Single Crystals}

A large (5-9 mm wide and $5 \mathrm{~cm}$ long) single crystal of TM60 was grown by the floating zone technique using a halogen lamp image furnace [Brice, 1986]. A feed rod of polycrystalline TM60 was synthesized by mixing hematite and titanium oxide powders in the appropriate proportions, cold pressing, and then sintering at approximately $1350^{\circ} \mathrm{C}$ in a reducing atmosphere for 24 hours.

In the image furnace, the feed rod is successively melted by mechanically translating it through the hot spot produced at the focal point of a gold plated ellipsoidal mirror containing two halogen lamps. Melting is initiated in the feed rod by gradually increasing the lamp power and the single crystal is then grown by recrystallizing the molten part of the feed rod onto a seed as the feed rod moves through the hot zone. The feed rod and seed are enclosed in a gas-tight quartz tube which allows the atmosphere to be controlled during crystal growth. Unlike the method of Brabers et al. [1984], this feature allows us to produce crystals with different "grown in" stoichiometries. We used a gas mix of $10 \mathrm{CO}_{2}: 1 \mathrm{CO}$ for TM60. Although the temperature inside the image furnace is not measured, the temperature gradient away from the focal point of the lamps is very steep. The feed rod and 
single crystal outside of the melt zone are nevertheless maintained at some elevated temperature due to thermal conduction of the gas in the quartz tube. Examination of the crystal using the optical microscope, scanning electron microscope, electron microprobe, thermomagnetic analysis, and Laue back-reflection camera shows it to be single crystal, single phase, and chemically homogeneous except for a small gradient in Ti/Fe along the growth dimension due to the different melt-solid distribution coefficients of these species. Two slices, one near the top (tc) and one towards the bottom (bc), separated by $4 \mathrm{~cm}$ along the growth direction were cut from the crystal. Chemical compositions obtained from electron microprobe analysis are $x=0.61$ and $x=0.58$ for tc and bc, respectively. Fifty separate spot analyses were made across the diameter of the samples and errors in the $x$-parameter were estimated to be \pm 0.01 . The resultant chemical gradient for the crystal of TM60 along the growth direction is $\approx 0.75 \mathrm{~mole} \% \mathrm{TV} / \mathrm{cm}$ and is negligible for the small (1-2 mm thick) samples used in the experiments.

\section{Magnetic Measurements}

\section{Curie Temperature}

Thermomagnetic curves for pieces from bc (first grown) and tc (last grown) are shown in Figure 1. Magnetization was measured in He gas using a vibrating sample magnetometer. The Curie temperatures $(T)$ are $229^{\circ} \mathrm{C}(\mathrm{bc})$ and $176^{\circ} \mathrm{C}(\mathrm{tc})$. This variation in $\mathrm{T}_{\mathrm{c}}$ is much greater than that expected for the small difference in composition and must be at least partly the result of a difference in the nonstoichiometry of the two samples [Hauptman, 1974]. Because the $\mathrm{fO}_{2}$ of the $1 / 10: \mathrm{CO} / \mathrm{CO}_{2}$ gas mix becomes increasingly more oxidizing relative to the TM60 stability field as temperature decreases, the earlier grown (bottom) part of the crystal may partially re-equilibrate to more oxidizing fugacities as it cools during continued growth of the crystal. Direct evidence of the effect of $\mathrm{fO}_{2}$ on $\mathrm{T}_{c}$ is provided by subsequently annealing a piece from bc in the same $1 / 10: \mathrm{CO} / \mathrm{CO}_{2}$ gas mix at $1300^{\circ} \mathrm{C}$ for $24 \mathrm{hrs}$., which caused $\mathrm{T}_{\mathrm{c}}$ to decrease to $203^{\circ} \mathrm{C}$ (sample ba in Fig. 1). Further annealing of ba at $1300^{\circ} \mathrm{C}$ just inside the

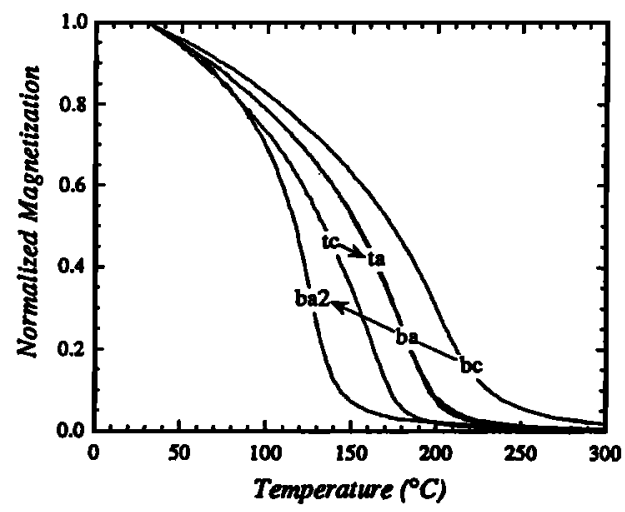

Figure 1. Normalized magnetization versus temperature for pieces from the top (tc, last grown) and bottom (bc, first grown) of a synthetic single crystal of TM60 "as grown." Samples ba and ba2 are pieces from be subsequently annealed at $1300^{\circ} \mathrm{C}$ for $24 \mathrm{hrs}$. at oxygen fugacities of $10^{-2.7}$ and $10^{-4} \mathrm{~Pa}$, respectively; ta is tc subsequently annealed at $1300^{\circ} \mathrm{C}$ for 24 hrs. at an $\mathrm{fO}_{2}=10^{-1.3} \mathrm{~Pa}$.

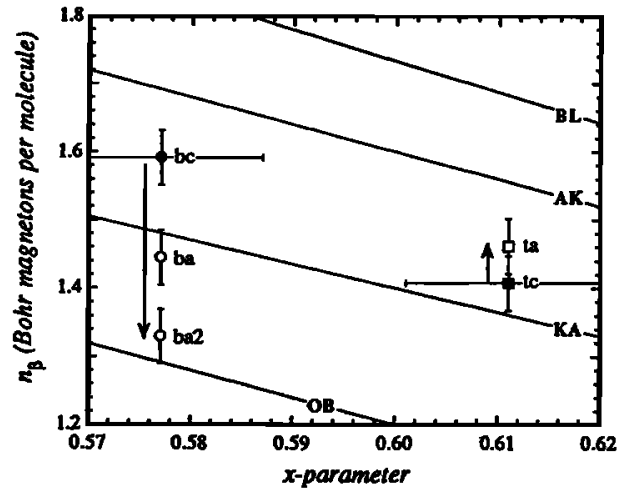

Figure 2. Magnetization $\left(n_{0}\right)$ versus composition $(x)$ for pieces of the synthetic TM60 crystal with different degrees of nonstoichiometry $(\delta)$ compared with that predicted by various cation distribution models. Samples bc, ba, ba2, tc, and ta are the same as in Fig. 1. Distribution models are BL (Bleil, 1971); AK (Akimoto, from O'Reilly, 1984); OB (O'Reilly and Banerjee, 1965); KA (Kakol et al., 1991).

reducing boundary of the TM60 stability field $\left(\mathrm{fO}_{2}=10^{-4} \mathrm{~Pa}\right)$ reduced $\mathrm{T}_{c}$ to $145^{\circ} \mathrm{C}$ (sample ba2 in Fig. 1). The decrease in $T_{c}$ correlates with the decrease in $\delta$ of the TM60 and is similar to that found by Hauptman [1974]. Oxidizing a piece from tc produced the opposite effect (sample ta in Fig. 1).

\section{Saturation Magnetization}

Magnetization measurements were made with a SQUID susceptometer (Quantum Design MPMS1) at $5 \mathrm{~K}$ in fields from 3 to $5.5 \mathrm{~T}$, after cooling from $300 \mathrm{~K}$ in a $5.5 \mathrm{~T}$ field. Samples were thin disks cut parallel to $\{100\}$ and oriented in the magnetometer so that the magnetic field was applied parallel to this plane, which contains the $<100\rangle$ easy axis of magnetization for TM60 [e.g., O'Reilly, 1984]. Saturation magnetization was calculated by extrapolating $\mathrm{H}^{-2}$ to infinite field. Although samples were not oriented with respect to their easy axes, the moments at $5.5 \mathrm{~T}$ were within $1 \%$ of the extrapolated value confirming that saturation was achieved. The moment at $5 \mathrm{~K}$ is plotted as a function of $x$ and $\mathrm{fO}_{2}$ of high temperature annealing in Figure 2 and compared with four cation distribution models for supposedly stoichiometric TM [O'Reilly and Banerjee, 1965; Bleil, 1971; O'Reilly, 1984; Kakol et al., 1991]. The moment $n_{\beta}$ decreases with decreasing $\mathrm{fO}_{2}$ (and $\delta$ ) during annealing.

The cation distribution problem in titanomagnetite consists of dividing $[(2-2 x)+2 \delta(3+x)] \mathrm{Fe}^{3+},[(1+x)-\delta(9+x)] \mathrm{Fe}^{2+}$, and $x(1-$ $\delta) \mathrm{T}^{4+}$ cations and $3 \delta$ vacancies between the $A$ and $B$ sites. Assuming simple A-B collinear magnetic interaction and Bsite only $\mathrm{Ti}^{4+}$ and vacancies [e.g., O'Reilly, 1984; Collyer et al., 1988] reduces the cation distribution problem to partitioning $\mathrm{Fe}^{3+}$ and $\mathrm{Fe}^{2+}$ between the $\mathrm{A}$ and $\mathrm{B}$ sites, and the saturation magnetization in Bohr magnetons per formula unit at absolute zero for known $\delta$ and $x$ is

$$
n_{\beta}=6(1-\delta)(1-x)-2 b
$$

Unlike the data shown in Figure 2, (3) predicts that, for constant $b, n_{\mathrm{p}}$ should decrease with increasing $\mathrm{fO}_{2}$ (and $\delta$ ) and that the magnitude of this decrease is $\sim 1 / 5$ that observed for the sample pair ba,ba2, even for the largest change in nonstoichiometry permitted $\left(\delta_{\max }=0.02\right)$ [Senderov et al., 
1993]. The changes in magnetization of the bottom piece as a result of the annealing and quenching are not consistent with previous hypotheses that faster quench rates or higher quench temperatures (samples ba and ba2) would lead to an Akimoto-like distribution and slower quench rates or lower quench temperatures (sample bc) to an O'Reilly-Banerjeelike distribution [Trestman-Matts et al., 1983; Stephenson, 1969; Bleil, 1971]. Instead, we hypothesize that the differences among the various cation distribution models result from differences in nonstoichiometry. At low temperatures, a decrease in nonstoichiometry apparently increases the degree of long range order (LRO) or, in other words, decreasing $\mathrm{fO}_{2}$ (and $\delta$ ) produces an apparent increase in $b$ and hence, a lower net magnetization in (3).

\section{Electrical Measurements}

Electrical transport properties in ferrospinels, including TM, are primarily determined by small polaron conduction between octahedral $\mathrm{Fe}$ ions [e.g. Wu and Mason, 1981]. Thermoelectric power $(\mathrm{Q})$, therefore, is a direct measure of the octahedral $\mathrm{Fe}^{3+} / \mathrm{Fe}^{2+}$ ratio [e.g., Wu and Mason, 1981] and can be used to obtain $b$ as a function of temperature and $\delta$

$$
Q \cong-\frac{k}{e} \ln \frac{2\left[F e^{3+}\right]}{\left[F e^{2+}\right]}=-\frac{k}{e} \ln \left(2 \frac{(2-2 x)+2 \delta(3+x)-b}{x+b-\delta(9+x)}\right)
$$

where $k$ is Boltzmann's constant and $e$ is electronic charge.

Thermopower was calculated from the slope of the EMF versus $\Delta \mathrm{T}$ as the sample was moved in the temperature gradient of the fumace using the experimental setup described by Duba et al. [1990]. The thermopower of samples from the top part of the TM 60 crystal was measured at $1200^{\circ} \mathrm{C}$ and oxygen fugacities of $10^{-3}, 10^{-4}$, and $10^{-5} \mathrm{~Pa}$ and is shown in Figure 3. Near the reduction boundary, our value for $\mathrm{Q}_{\mathrm{TM} 60} \approx-44 \mu \mathrm{V} / \mathrm{deg}$ agrees well with the results of TrestmanMatts et al. [1983] and suggests that, using the accepted site occupancy model in (1), the inverse-like cation distribution $(b \rightarrow 1)$ of TM60 at low temperature changes toward a more normal-like distribution $(b \rightarrow 0)$ at high temperatures. In addition, we find a small but reproducible and reversible decrease in $\mathrm{Q}$ with increasing oxygen fugacity above $10^{-4} \mathrm{~Pa}$ at $1200^{\circ} \mathrm{C}$. As written, (4) is appropriate for B-site only hopping and vacancies and requires a decrease in the $\left[\mathrm{Fe}^{3+}\right] /$ $\left[\mathrm{Fe}^{2+}\right]$ on the B-site to explain the observed decrease in ther-

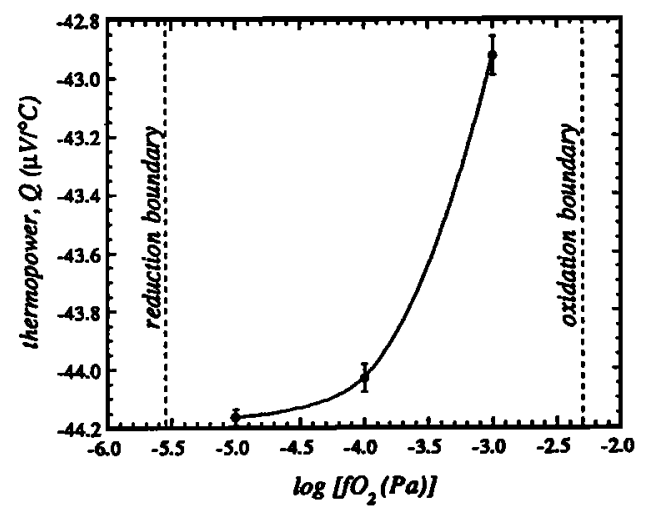

Figure 3. Thermopower $(Q)$ versus $\log \mathrm{fO}_{2}$ for the synthetic TM60 crystal at $1200^{\circ} \mathrm{C}$.

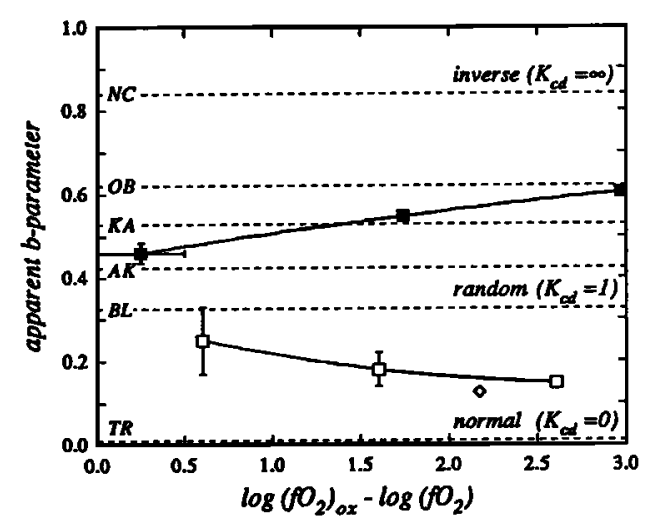

Figure 4. Apparent $b$ calculated from the low temperature magnetization (solid symbols) and high temperature thermopower measurements (open symbols) versus relative oxidation defined as the difference between the $\mathrm{fO}_{2}$ of the oxidation boundary of the TM60 crystal and the $\mathrm{fO}_{2}$ imposed during high temperature annealing. The open diamond is calculated from the thermopower extrapolated to $1200^{\circ} \mathrm{C}$ for supposedly stoichiometric TM60 [Trestman-Matts et al., 1983]. The cation distribution models shown are: NC (NéelChevallier), BL (Bleil), AK (Akimoto), OB (O'Reilly-Banerjee), KA (Kakol), and TR (Trestman-Matts et al., 1984).

mopower, even though $\left[\mathrm{Fe}^{3+}\right]$ in the crystal as a whole should increase with increasing $\mathrm{fO}_{2}$. Although a more complicated expression than (4) is necessary should these constraints be relaxed, the thermopower measurements corroborate that $b$ is sensitive to changes in nonstoichiometry and exhibits an apparent increase with increasing $\delta$ at high temperature.

\section{Discussion and Summary}

The $\mathrm{fO}_{2}$-dependence of $\mathrm{T}_{c}$ is similar to that obtained by Hauptman [1974] who also calculated $\delta$ as a function of $\mathrm{fO}_{2}$ after quenching. Recent estimates of $\delta$ as a function of $\mathrm{fO}_{2}$ by Senderov et al. [1993] are similar to those of Hauptman [1974] and suggest much higher degrees of nonstoichiometry in Ti-rich TM than previously hypothesized by Aragon and McCallister [1982], who assumed that vacancies are restricted to the magnetite component of TM.

The apparent changes in $b$ as a function of $\mathrm{fO}_{2}$ for both the magnetic and electrical measurements assuming only B-site vacancies and octahedral hopping are summarized in Figure 4. The high temperature $b$ parameters are the average of a lower limit (using $\delta=0$ ) and an upper limit (using $\delta$ estimated from Senderov et al. [1993]) calculated from the thermopower data using (4). The low temperature $b$ parameters are calculated in a similar manner from the magnetization data using (3). In terms of the cation distribution models shown in Figure 4, $K_{c d}=\infty$ corresponds to the Neel-Chevallier model; $K_{c c}=1$ corresponds to the Bleil [1971] model; and, $K_{c d}=0$ corresponds to the high temperature distribution of TrestmanMatts et al. [1983]. If it is further assumed that B-sites can accept a maximum of one $\mathrm{Fe}^{3+}$ ion, then $K_{c d}=1$ corresponds to the Akimoto model. The O'Reilly-Banerjee and Kakol models arise from $1<K_{e d}<\infty$. Both the low temperature and high temperature measurements indicate that the cation distribution of TM60 becomes more random with increasing 
oxidation/nonstoichiometry. At the low temperatures for which these models were developed, the O'Reilly-Banerjee model closely approximates $b$ determined for the single crystals under reducing conditions while the Akimoto model is a better approximation under oxidizing conditions. If Asite vacancies are allowed, the apparent changes in $b$ with increasing oxidation are smaller than shown in Figure 4, reducing the apparent increase in $b$ calculated from (4) by $10 \%$ at most and canceling or even reversing the apparent decrease in $b$ calculated from (3).

The relative changes in $b$ at low and high temperatures as a function of oxidation suggest that cation vacancies stabilize a more random cation distribution than would otherwise be obtained for stoichiometric or reduced TM60. The production of cation vacancies must be compensated by the production of an appropriate number of positively charged species, for example, $\mathrm{Fe}^{3+}$. If vacancies are more easily accommodated on A sites at high temperatures, they may also be accompanied by a greater than normal concentration of $\mathrm{Fe}^{3+}$ on A sites (higher $b$ ) either for local charge balance or due to defect association. At low temperatures, these vacancies would "return" with their accompanying $\mathrm{Fe}^{3+}$ to $\mathrm{B}$ sites, resulting in a lower $b$ value relative to reduced TM60. This model requires electron hopping between $A$ and $B$ sites and that cation vacancy diffusion between $A$ and $B$ sites in the same unit cell is rapid enough to prevent complete quenching of the high temperature distribution of these defects. The differences among the various experimental cation distribution models may thus be explained by a previously unaccounted for variation in nonstoichiometry of the samples used by the different research groups.

The data presented above underscore the discrepancies between theoretical models of the cation and vacancy distributions in titanomagnetite and those necessary to explain our experimental measurements of electrical and magnetic behavior. Our preliminary single crystal data show that changes in nonstoichiometry may have a profound influence on LRO and resulting magnetic behavior of TM60 and titanomagnetites in general. Further measurements of the electrical and magnetic properties of titanomagnetites under strictly controlled thermodynamic conditions are necessary to construct a model more compatible with the experimental data and test the hypotheses suggested by our preliminary work.

Acknowledgments. The single crystal used in these experiments was grown using the Nichiden Machinery, Ltd. arc image furnace (model SC-35HD) maintained by the Department of Earth Sciences at Lawrence Livermore National Laboratory. Electrical experiments were also performed at LLNL through the generosity and assistance of Dr. Al Duba. The authors gratefully acknowledge reviews by T. Mason and Ö. Özdemir. This research is supported by NSF contracts EAR-901789 and EAR9219621 and is contribution 9401 of the Institute for Rock Magnetism. The IRM is funded by grants from the Keck and National Science Foundations and the University of Minnesota.

\section{References}

Aragon, R. and R. H. McCallister, Phase and point defect equilibria in the titanomagnetite solid solution, Phys. Chem. Min., 8, 112-120, 1982.

Bleil, U., Cation distribution in titanomagnetites, Zeitschrift fur Geophysik, 37, 305-319, 1971.

Brabers, V. A. M., T. E. Whall, and P. S. A. Knapen, Preparation of polycrystalline and monocrystalline $\mathrm{Fe}_{3-x} \mathrm{Ti}_{x} \mathrm{O}_{4}$ spinels, J. Crystal Growth, 69, 101-107, 1984.

Brice, J.C., Crystal Growth Processes, Blackie, Glasgow and London, 298 pp., 1986.

Collyer, S., N. W. Grimes, D. J. Vaughan, and G. Longworth, Studies of the crystal structure and crystal chemistry of titanomaghemite, Amer. Mineral., 73, 153-160, 1988.

Dieckmann, R., Defects and cation diffusion in magnetite (IV): Nonstoichiometry and point defect structure of magnetite ( $\left.\mathrm{Fe}_{3-5} \mathrm{O}_{4}\right)$, Ber. Bunsenges. Phys. Chem., 86, 112-118, 1982.

Duba, A.G., R.N. Schock, E.L. Arnold, and T.J. Shankland, An apparatus for measurement of electrical conductivity to $1500^{\circ}$ $\mathrm{C}$ at known oxygen fugacity, in The Brittle-Ductile Transition in Rocks, Geophys. Monogr. Ser., vol. 56, ed. by A.G. Duba et al., pp. 207-210, AGU, Washington, D.C., 1990.

Hauptman, Z., High temperature oxidation, range of nonstoichiometry and Curie point of cation deficient titanomagnetite $\mathrm{Fe}_{2.4} \mathrm{Ti}_{0.6} \mathrm{O}_{4+r}$ Geophys. J. $R$. astron. Soc., 38, 29-47, 1974.

Kakol, Z., J. Sabol, and J. M. Honig, Cation distribution and magnetic properties of titanomagnetites $\mathrm{Fe}_{3-x} \mathrm{Ti}_{x} \mathrm{O}_{4}(0 \leq x<1)$, Phys. Rev. B, 43, 649-654, 1991.

Lindsley, D.H. (Ed.), Oxide Minerals: Petrologic and Magnetic Significance, Rev. Mineral, 25, 509 pp., MSA, Washington, D.C., 1990.

Moskowitz, B. M., Towards resolving the inconsistencies in characteristic physical properties of synthetic titanomaghemites, Phys. Earth Planet. Int., 46, 173-183, 1987.

O'Neill, $H$. and A. Navrotsky, Cation distributions and thermodynamic properties of binary spinel solid solutions, Am. Mineral, 69, 733-753, 1984.

O'Reilly, W. and S.K. Banerjee, Cation distribution in titanomagnetites (1-x) $\mathrm{Fe}_{3} \mathrm{O}_{4} \times \mathrm{Fe}_{2} \mathrm{TiO}_{4}$, Phys. Lett., 17, 237 238, 1965.

O'Reilly, W., Rock and Mineral Magnetism, Blackie, Glasgow, 222 pp., 1984.

Senderov, E., A. U. Dogan, and A. Navrotsky, Nonstoichiometry of magnetite-ulvőspinel solid solutions quenched from $1300^{\circ} \mathrm{C}$, Amer. Mineral., 78, 565-573, 1993.

Stephenson, A., The temperature dependent cation distribution in titanomagnetites, Geophys. J. R. astron. Soc., 18, 199-210, 1969.

Trestman-Matts, A., S.E. Dorris, S. Kumarakrishnan, and T.O. Mason, Thermoelectric determination of cation distribution in $\mathrm{Fe}_{3} \mathrm{O}_{4}-\mathrm{Fe}_{2} \mathrm{TiO}_{4}$, J. Amer. Cer. Soc., 66, 829-834, 1983.

Wechsler, B. A., D. H. Lindsley, and C. T. Prewitt, Crystal structure and cation distribution in titanomagnetites $\left(\mathrm{Fe}_{3-\mathrm{x}}\right.$ $\mathrm{Ti}_{\mathrm{K}} \mathrm{O}_{4}$ ), Amer. Mineral., 69, 754-770, 1984.

Wu, C. C. and T. O. Mason, Thermopower measurement of cation distribution in magnetite, J. Amer. Cer. Soc., 64, 520$522,1981$.

B. Wanamaker and B. Moskowitz, Dept. of Geology and Geophysics, 310 Pillsbury Dr., S.E., University of Minnesota, Minneapolis, MN 55455. (e-mail: bwana@maroon.tc.umn.edu)

(Received February 7, 1994; accepted March 4, 1994.) 